\title{
OPEN Dual-functional ultraviolet photodetector with graphene electrodes on AlGaN/GaN heterostructure
}

\author{
Bhishma Pandit ${ }^{1}$, E. Fred Schubert ${ }^{2} \&$ Jaehee Cho ${ }^{1 凶}$
}

A dual-functional ultraviolet (UV) photodetector with a large UV-to-visible rejection ratio is presented, in which interdigitated finger-type two-dimensional graphene electrodes are introduced to an $\mathrm{AlGaN} / \mathrm{GaN}$ heterostructure. Two photocurrent generation mechanisms of photovoltaic and photoconductive dominances coexist in the device. The dominance of the mechanisms changes with the induced bias voltage. Below a threshold voltage, the device showed fairly low responsivities but fast response times, as well as a constant photocurrent against the induced bias. However, the opposite characteristics appeared with high bias voltage. Specifically, above the threshold voltage, the device showed high responsivities with additional gain, but slow rise and recovery times. For instance, the responsivity of $10.9 \mathrm{~A} / \mathrm{W}$ was observed with the gain of 760 at the induced bias voltage of $5 \mathrm{~V}$. This unique multifunctionality enabled by the combination of an $\mathrm{AIGaN} / \mathrm{GaN}$ heterostructure with graphene electrodes facilitates the development of a single device that can achieve multiple purposes of photodetection.

Semiconductor-based ultraviolet (UV) photodetectors with very high sensitivity, stability, speed, and signal-tonoise $(\mathrm{S} / \mathrm{N})$ ratio have attracted much attention in recent years for their potential applications in environmental monitoring and security systems. Such applications include flame monitoring, missile plume detection, ozone layer monitoring, biological and chemical analysis, and secure optical communication in outer space ${ }^{1-4}$. As demand and expectation have begun to exceed the performance of conventional UV photodetectors, the development of novel UV photodetectors with special functions or multiple functions has become necessary ${ }^{5,6}$. Smart, flexible, and self-powering characteristics, as well as intelligence, are expected to be essential for next-generation UV photodetectors in the internet-of-things (IoT) era ${ }^{7,8}$.

In order to develop high-performance UV photodetectors, various material- and structure-based approaches have been investigated and published ${ }^{2,4,9,10}$. First, regarding materials, $\mathrm{GaN}$ and its alloys with $\mathrm{Al}$ and In are highly promising for both visible-blind and solar-blind UV detection without requiring bulky optical filters or cooling systems, unlike Si-based UV photodetectors ${ }^{11}$. Special attention has been paid to AlGaN ternary semiconductors, in which, by tuning the $\mathrm{Ga}$ and $\mathrm{Al}$ compositions, the bandgap energy can be varied from $3.4 \mathrm{eV}(\mathrm{GaN})$ to $6.2 \mathrm{eV}(\mathrm{AlN})$, thereby covering the UVA to UVC spectral region. In addition to the use of a single layer of nitride semiconductors for photodetector applications, an $\mathrm{AlGaN} / \mathrm{GaN}$ heterostructure can provide additional benefits of high-speed and high-gain operation. Such a heterostructure intrinsically forms a two-dimensional electron gas (2DEG) at the interface between AlGaN and GaN; this simultaneously provides both high carrier density and mobility ${ }^{12-14}$. In fact, $\mathrm{AlGaN} / \mathrm{GaN}$ heterostructures are essential platforms for high-electron-mobility transistor technology for next-generation high-power and high-frequency devices ${ }^{15,16}$.

Graphene, a two-dimensional (2D) hexagonal lattice array of carbon atoms, is attractive for applications as transparent conductive electrodes because of its high electrical and thermal conductivities, transparency, and mechanical strength ${ }^{17-20}$. Previous studies have confirmed that the graphene/GaN contact is stable under hightemperature and high-radiation operations, indicating that graphene electrodes are appropriate for applications in fire alarms and space exploration devices, which operate in extremely harsh conditions ${ }^{21}$. Our previous studies demonstrated the successful implementation of graphene and reduced graphene oxide as transparent electrodes on AlGaN layers with various Al mole fractions, showing sharp cut-off wavelengths at the energy bandgaps of

${ }^{1}$ School of Semiconductor and Chemical Engineering, Jeonbuk National University, Jeonju 54896, Republic of Korea. ${ }^{2}$ Department of Electrical, Computer, and Systems Engineering, Rensselaer Polytechnic Institute, Troy, NY 12180, USA. ${ }^{\boxplus}$ email: jcho@jbnu.ac.kr 

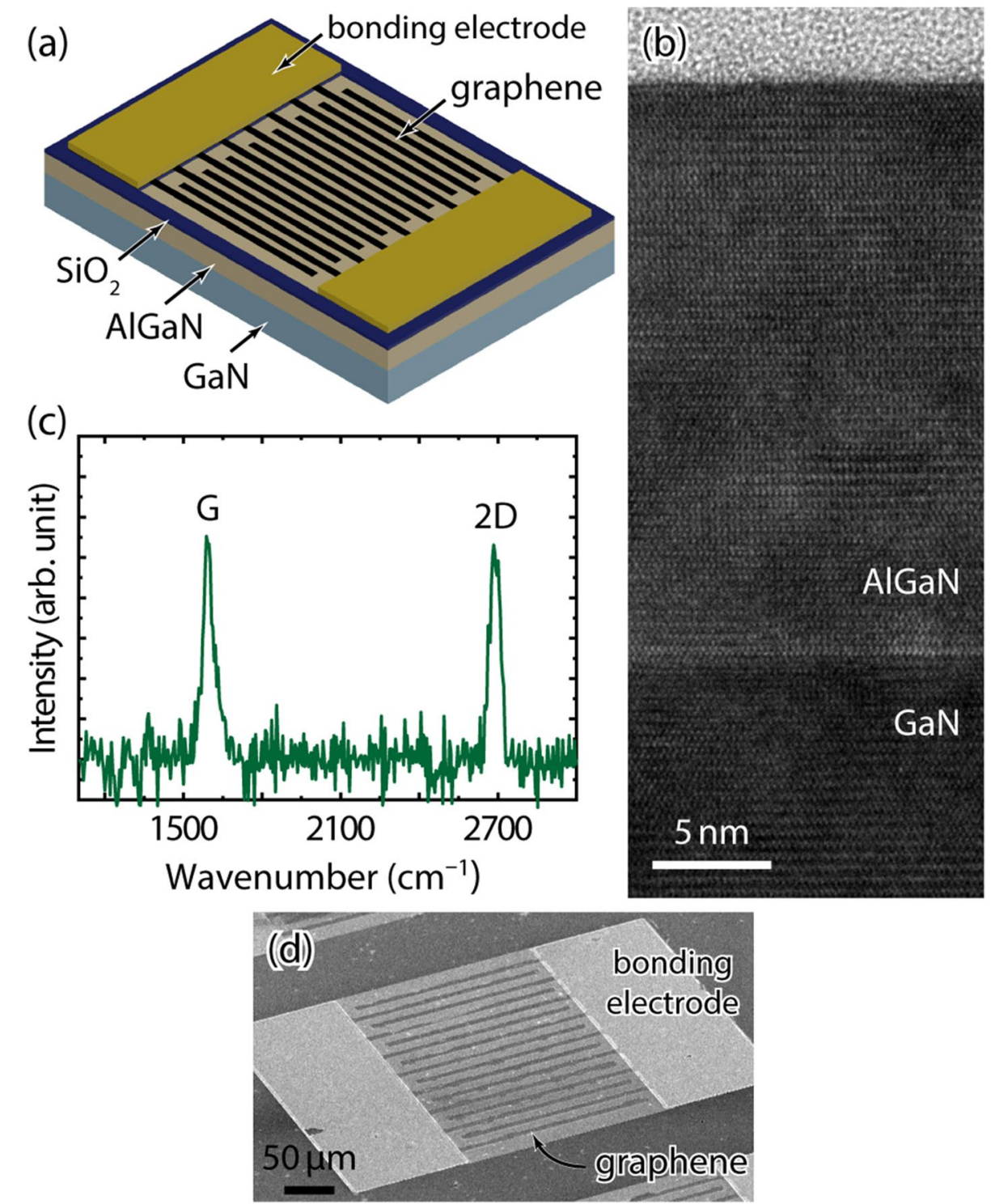

Figure 1. Structural analysis. (a) Schematic of the Gr/AlGaN/GaN photodetector structure. (b) Transmission electron microscope image of $\mathrm{AlGaN} / \mathrm{GaN}$ heterostructure. (c) Raman spectrum of $\mathrm{Gr} / \mathrm{AlGaN} / \mathrm{GaN}$. (d) Scanning electron microscope image of the fabricated device.

$\mathrm{AlGaN}^{22,23}$. In this regard, the combination of graphene electrodes and $\mathrm{AlGaN} / \mathrm{GaN}$ heterostructures may yield an excellent UV photodetector, considering the high transparency of graphene in the UV spectral region and the high electrical conductivities of graphene and 2DEG, which would benefit efficient charge collection in the photodetector.

Secondly, in structural approaches, various photodetector structures have been employed, including a metal-semiconductor-metal (MSM) photodiode, $\mathrm{p}-\mathrm{n}$ and $\mathrm{p}-\mathrm{i}-\mathrm{n}$ photodiodes, an avalanche photodiode, a photoconductor, and a phototransistor ${ }^{4,24}$. Among them, the MSM structure with back-to-back Schottky contacts provides a low dark current, high $\mathrm{S} / \mathrm{N}$ level, fast response speed, and compatibility for integration with other circuit components, particularly via simple fabrication methods ${ }^{25,26}$. The MSM-type structure can be fabricated in one step of electrode deposition on a semiconductor material without any deteriorations of device performance from the complicated processes of etching and diffusion, as well as those from the asymmetric traveling lengths of electrons and holes before reaching an electrode.

In this study, we present a dual-functional (i.e., either high-speed or high-gain) UV photodetector comprising an $\mathrm{AlGaN} / \mathrm{GaN}$ heterostructure and a graphene electrode (hereafter, Gr/AlGaN/GaN). The photodetectors presented here have an MSM-type structure, as shown schematically in Fig. 1a, and were investigated under broad wavelength range illumination and various bias voltages. We found that two photocurrent generation mechanisms of photovoltaic and photoconductive work together in the devices; these dual functionalities can be easily switched by varying the induced bias voltage on the device. 


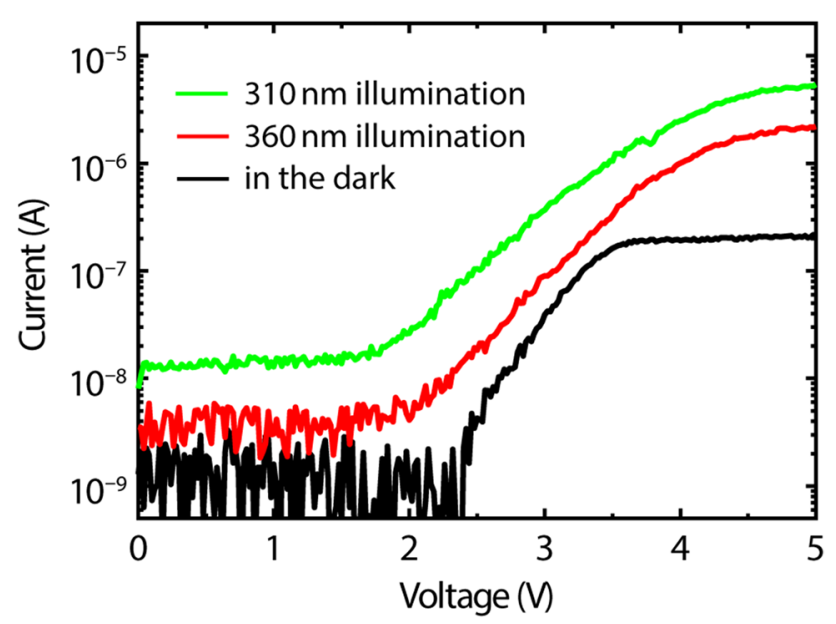

Figure 2. Current-voltage characteristics of the $\mathrm{Gr} / \mathrm{AlGaN} / \mathrm{GaN}$ photodetector under various illumination conditions.

\section{Results}

The transmission electron microscopy (TEM) image in Fig. 1b shows a strain-unrelaxed epitaxially grown AlGaN layer on the thick GaN underlayer. The growth of the AlGaN/GaN heterostructure spontaneously initiated the formation of a $2 D E G$ with the mobility of $1980 \mathrm{~cm}^{2} / \mathrm{V} \cdot \mathrm{s}$ and the sheet carrier density of $5.6 \times 10^{12} \mathrm{~cm}^{-2}$ at room temperature. Figure 1c shows the Raman spectroscopic profile of the Gr/AlGaN/GaN. The well-known G and 2D peaks of graphene are found at the wavenumbers of about 1590 and $2700 \mathrm{~cm}^{-1}$, respectively ${ }^{27}$. The peak intensity ratio of $\mathrm{I}_{2 \mathrm{D}} / \mathrm{I}_{\mathrm{G}}$ is less than 1 , indicating the presence of multilayered graphene ${ }^{28}$. The final MSM-like device structure of $\mathrm{Gr} / \mathrm{AlGaN} / \mathrm{GaN}$ stacks has eight pairs of interdigitated finger electrodes of $10 \mu \mathrm{m}$ in width, $10 \mu \mathrm{m}$ in spacing, and $300 \mu \mathrm{m}$ in length, as shown in the scanning electron microscope image of Fig. $1 \mathrm{~d}$.

Figure 2 shows the current-voltage (I-V) characteristics of a Gr/AlGaN/GaN photodetector in the dark and under illumination at 360 and $310 \mathrm{~nm}$. A close examination of the I-V curve in the dark (black line in Fig. 2) shows typical diode behavior, i.e., an extremely low dark current of $\sim 1 \mathrm{nA}$ below a threshold voltage, a gradual increase in current above the threshold voltage, and then current saturation due to the electron saturated velocity. Because of the Schottky-like contact property between graphene and AlGaN (see "Supplementary Information" for more details on the Schottky barrier height), a threshold voltage of about $2.4 \mathrm{~V}$ exists before current increases exponentially as a function of bias. As shown in Fig. 2, photocurrents are generated under illumination at both 360 and $310 \mathrm{~nm}$, with distinct photoresponsivity over the entire bias voltage range. The shorter incident wavelength yields a higher photocurrent with faster turn-on (i.e., a lower threshold voltage). Absorption of the high-energy photons may lower the energy barrier of the Schottky-like contact, thus lowering the threshold voltage. Overall, the shapes and tendencies of the three I-V curves are similar, except current saturation does not occur for the photocurrents under 360- and 310-nm illumination.

As a representative figure-of-merit of a photodetector, the responsivity (R) was calculated by using the relation ${ }^{29}$ :

$$
R=\left(I_{P}-I_{D}\right) / \Phi
$$

where $I_{D}$ is the dark current, $I_{P}$ is the photocurrent, and $\Phi$ is the incident optical power, all of which are easily measurable values. Measurements of responsivity as a function of wavelength are performed at various bias conditions, as shown in Fig. 3a. Each point is the average value of measurements from multiple devices. These data demonstrate a high UV-to-visible rejection ratio, showing low responsivity in the visible region but increasing responsivity as the incident wavelength enters the UV spectral region, with an exponential increase with further decreases in wavelength. Another important observation in Fig. $3 \mathrm{a}$ is the dependency of the responsivity on the induced bias. At low bias voltages (i.e., 1 and $2 \mathrm{~V}$ ), the responsivities have low absolute values; indeed, they are below the theoretical limits of responsivity for devices in the photovoltaic regime. Details of the two different photocurrent generation mechanisms of a photodetector follow in the next paragraph. In order to examine the theoretical limit of responsivity in detail, the responsivity of Eq. (1) is rewritten as follows ${ }^{29}$ :

$$
R=\eta q / h v \approx \eta \lambda / 1.24
$$

where $\eta$ is the quantum efficiency, $\mathrm{q}$ is the elementary charge, $\mathrm{h}$ is Planck's constant, and $v$ and $\lambda$ are respectively the frequency in $\mathrm{Hz}$ and wavelength in $\mu \mathrm{m}$. According to this equation, $\mathrm{R}$ has a theoretical limit (i.e., $\mathrm{R}$ at $\eta=100 \%)$ at each wavelength, which can be reached when the device involves no gain mechanism. The dotted line in Fig. 3a shows the limit as a function of the wavelength; the responsivities of the device when operated at 1 and $2 \mathrm{~V}$ are always below this line. However, the responsivities at the bias voltages of 3 and $4 \mathrm{~V}$ are higher than those at 1 and $2 \mathrm{~V}$, and they exceed the theoretical limit at the short-wavelength region. For example, under $310-\mathrm{nm}$ illumination, the device has significantly higher responsivities of 0.8 and $4.5 \mathrm{~A} / \mathrm{W}$ at the bias voltages 

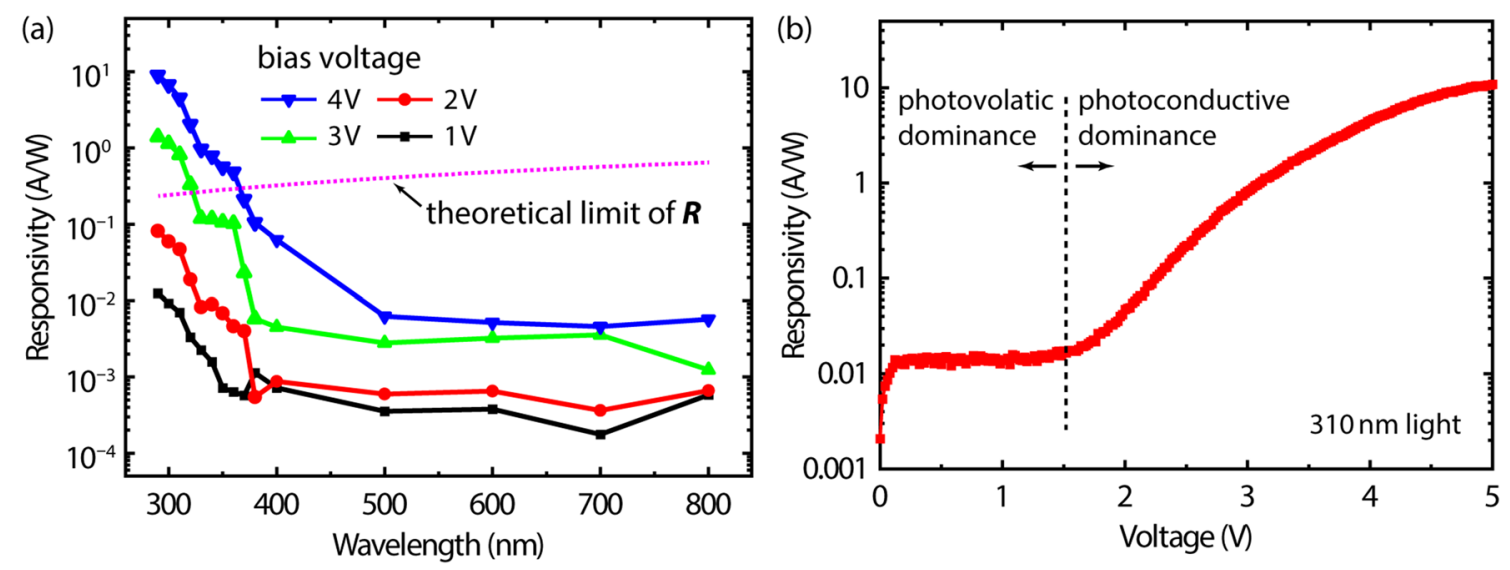

Figure 3. Photoresponse. (a) Spectral photoresponse of the Gr/AlGaN/GaN photodetector under various bias voltages. (b) Responsivity as a function of induced voltage under 310-nm illumination. Note the logarithmic scale of the responsivity axis.

of 3 and $4 \mathrm{~V}$, respectively, in contrast to those of 0.01 and $0.05 \mathrm{~A} / \mathrm{W}$ at the bias voltages of 1 and $2 \mathrm{~V}$, respectively. The higher responsivities exceed that indicated by the $100 \%$ quantum efficiency limit of $0.25 \mathrm{~A} / \mathrm{W}$ at $310 \mathrm{~nm}$. Therefore, these high responsivities indicate the presence of a gain mechanism in the photodetector device.

Now let us discuss two different photocurrent generation mechanisms that can exist in semiconductor photodetectors ${ }^{29}$. First, a junction of two dissimilar materials can yield the photovoltaic effect, in which the dissimilarity can be different dopant types (homojunction), different materials themselves (heterojunction), and the contact between metal and semiconductor (Schottky-type) ${ }^{4,30}$. As photons impinging on the junction are absorbed, the absorbed photons produce free charge carriers within the depletion region. The existing electric field then causes the charge carriers to move apart, resulting in current flow in an external circuit. The most common photovoltaic-effect-based photodetectors are photodiodes and phototransistors. Second, photoconductivity (i.e., the photoconductive effect) occurs when photons impinge on semiconducting materials, causing the excitation of electrons from the valence band to the conduction band, and thus affecting the material conductivity ${ }^{31,32}$. This photoconductive effect is widely utilized in electromagnetic radiation sensors, photoconductors, light-dependent resistors, and photoresistors. CdS and CdSe are typical semiconductors used in photoconductive-effect-based photodetectors and sensors ${ }^{33,34}$. Generally speaking, photodetectors in these two categories have certain strengths and weaknesses. For example, in comparing a photodiode and a photoconductor, a photodiode operated under the photovoltaic effect has device characteristics as follows ${ }^{35,36}$ : (1) relatively low responsivity limited by the wavelength [refer to Eq. (2)], (2) high detectivity, (3) fast response time, and (4) ideally no dependence of responsivity on external bias. However, a photoconductor operated under the photoconductive effect has the opposite device characteristics, as follows: (1) relatively high responsivity due to an internal gain process, (2) low detectivity, (3) slow response time, and (4) strong dependence of responsivity on external bias. If only the respective strengths of these two device types could be combined, the resulting device would be great for diverse and versatile photodetectors.

Figure $3 \mathrm{~b}$ shows the responsivity of a typical $\mathrm{Gr} / \mathrm{AlGaN} / \mathrm{GaN}$ photodetector as a function of the bias voltage under 310-nm illumination. At low bias voltage $(<1.5 \mathrm{~V})$, the responsivity is nearly constant (e.g., $0.014 \mathrm{~A} / \mathrm{W}$ at $1 \mathrm{~V}$ ) with a weak dependency on bias voltage, which is an obvious indication that the device is operating in a manner dominated by the photovoltaic effect. Above $1.5 \mathrm{~V}$, the responsivity begins to increase, reaching $10.9 \mathrm{~A} / \mathrm{W}$ at the bias voltage of $5 \mathrm{~V}$, thus showing a strong dependency on the bias voltage. Note that signs of the two different photocurrent generation mechanisms aforementioned exist in a single device, revealing their specific device characteristics based upon the induced bias conditions. This dual functionality is enabled by the combination of the $\mathrm{AlGaN} / \mathrm{GaN}$ heterostructure and 2D graphene electrode. Graphene typically forms a Schottky contact on AlGaN, but the Schottky barrier height, or the energy barrier between graphene and AlGaN, is not stiff because of the inhomogeneity of Schottky barriers and the surface-state pinning; therefore, the barrier is easily overcome by the induced bias voltage. Meanwhile, the $2 \mathrm{DEG}$ of the $\mathrm{AlGaN} / \mathrm{GaN}$ heterostructure provides a superior platform to multiply charge carriers excited by light absorption. In other words, the photovoltaic regime is maintained as long as the Schottky-like contact of graphene/AlGaN is valid. After the energy barrier of this contact is punched through at the threshold voltage, the current path from one graphene electrode to the AlGaN/GaN heterojunction via $2 \mathrm{DEG}$ and then to the other graphene electrode behaves like a conductor. Therefore, a photocurrent gain $(G)$ occurs and works to increase the primary photocurrent, implying the shift from the photovoltaic to photoconductive regime. Equation (1) can be rearranged to express $I_{P}$ with $G$ as follows ${ }^{29}$ :

$$
I_{P}-I_{D}=R \Phi G \approx R \Phi \tau / t_{n}
$$

where $\tau$ is the minor-carrier lifetime and $t_{n}$ is the major-carrier transit time. Here, $t_{n}$ is inversely proportional to the induced bias voltage as $t_{n}=l /\left(\mu_{e} E\right)$, where $l$ is the photoconductor length, $E$ is the electric field, and $\mu_{\mathrm{e}}$ is the electron drift mobility, showing a high dependency on bias. For instance, a simple calculation comparing the photocurrents at 1 and $5 \mathrm{~V}$ shows that the gain of the device increases to 790 under 310 -nm illumination as the 

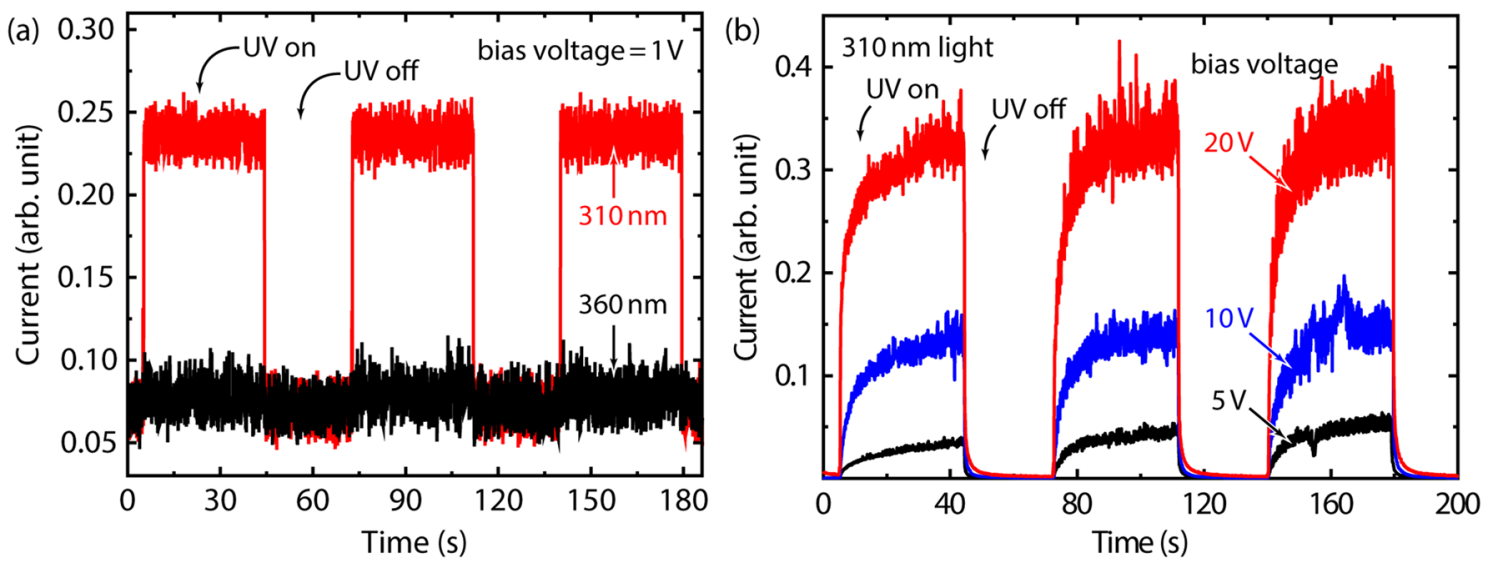

Figure 4. Temporal photoresponse of the $\mathrm{Gr} / \mathrm{AlGaN} / \mathrm{GaN}$ photodetector (a) at the bias voltage of $1 \mathrm{~V}$ under 310- and 360-nm illumination, (b) at the bias voltages of 5, 10, and $20 \mathrm{~V}$ under 310-nm illumination.

induced bias voltage is changed from 1 to $5 \mathrm{~V}$. The value is comparable to that reported previously by Satterthwaite et al. with an MSM-type photodetector comprising an AlGaN/GaN heterostructure and a Pd/Au electrode ${ }^{37}$.

The response speed of a photodiode is mainly limited by the carrier transit time in the depletion region, if the external effects such as capacitances and external resistors are excluded from consideration. On the other hand, the response speed of a photoconductive detector is influenced not only by the carrier transit time, but also by the minor-carrier lifetime. The longer carriers live, the higher they contribute to photocurrent; however, a long carrier lifetime consequently leads to a slow response speed. Therefore, for strong evidence of the coexistence of the two photocurrent mechanisms in one device, the temporal photoresponses were measured while changing the induced bias voltage. Figure 4a shows the temporal photoresponse of the Gr/AlGaN/GaN photodetector at $1 \mathrm{~V}$ with 360- and 310-nm illumination. Note that the bias condition of $1 \mathrm{~V}$ is part of the photovoltaic regime, as shown in Fig. 3b. No photoresponse occurs under 360-nm illumination, but the data are shown for comparison purposes. Under 310-nm illumination, a sharp on/off effect in the photocurrent is observed, with response and recovery times of $<0.1 \mathrm{~s}$. For high induced bias voltages, Fig. $4 \mathrm{~b}$ shows the temporal photoresponses measured under 310-nm illumination. The higher bias voltages from 5 to $20 \mathrm{~V}$ show significantly larger photocurrents, confirming the strong dependency on bias voltage. Contrary to the result in Fig. 4a, the photoresponses at the bias voltages of 5, 10, and $20 \mathrm{~V}$ show slow gradual rises and recoveries against on/off operation. Again, these slow photodetector responses are characteristic of the photoconductive regime, including the 5-, 10-, and 20-V bias conditions. Temporal measurements at low- and high-bias conditions thus represent well the specific characteristics of the photovoltaic and photoconductive effects, verifying that two different photocurrent generation mechanisms occur in the device, which can be selected by the induced bias voltage.

As another key parameter of photodetectors, the detectivity (D) shows how much minimum signal can be detected by the photodetector. The detectivity can be calculated by using the simplified equation ${ }^{38,39}$ :

$$
D \approx \frac{A^{\frac{1}{2}} R}{\left(2 q I_{D}\right)^{\frac{1}{2}}}
$$

where $\mathrm{A}$ is the junction area and the other symbols have their usual meanings as previously mentioned. The calculation showed the detectivities of $1.02 \times 10^{10}$ and $6.19 \times 10^{11} \mathrm{cmHz}^{1 / 2} \mathrm{~W}^{-1}$ at the bias voltages of 1 and $4 \mathrm{~V}$, respectively, under $310 \mathrm{~nm}$ (see "Supplementary Information", Table S1). Although the detectivities in the photoconductive regime show higher values than those in the photovoltaic regime, the increase in detectivity is not comparable with the increase in responsivity because the large dark current in the photoconductive regime.

\section{Discussion}

In conclusion, a dual-functional Gr/AlGaN/GaN UV photodetector with a clear UV-to-visible rejection ratio is presented. The photodetector shows two photocurrent generation mechanisms in a single device; the mechanisms can be selected by the induced bias voltage. Under low bias conditions, i.e., below the threshold voltage, the device showed relatively low responsivities, but fast response times and constant photocurrent against the induced bias. At high-bias conditions, i.e., above the threshold voltage, the device showed high responsivities with additional gain, but a slow response time and a strong dependency of the photocurrent on the induced bias. Depending on the bias voltage induced on the photodetector device, the dominant device features of the photodetector can be easily tuned; thus, a single device can be adjusted to meet any purpose of UV photodetection. This unique multifunctionality enabled by the combination of an $\mathrm{AlGaN} / \mathrm{GaN}$ heterostructure and a $2 \mathrm{D}$ graphene electrode is expected to be highly useful to the development of UV photodetectors for the coming IoT era. 


\section{Methods}

Device fabrication. Devices were fabricated on an AlGaN/GaN heterostructure grown on a Si substrate by metalorganic chemical vapor deposition (MOCVD). In detail, after a 5- $\mu \mathrm{m}$-thick layer of undoped $\mathrm{GaN}$ underlayer was grown on a p-type 6-in. $\mathrm{Si}$ (111) substrate, a 21-nm-thick AlGaN layer with a $20 \% \mathrm{Al}$ mole fraction and a 3-nm-thick GaN cap layer were subsequently grown on the GaN underlayer. Multilayered CVD-grown graphene was transferred by a polymethyl methacrylate (PMMA)-assisted wet transfer method ${ }^{40}$. In brief, PMMA was spin-coated on a graphene-coated $\mathrm{Cu}$ foil and subjected to soft baking at $180{ }^{\circ} \mathrm{C}$ for $5 \mathrm{~min}$. The PMMA/ graphene/Cu stack was then treated with ammonium persulfate solution for $7 \mathrm{~h}$ to etch away $\mathrm{Cu}$. In sequence, the separated graphene/PMMA stack was transferred to the surface of the AlGaN/GaN heterostructure sample. To improve the adhesion between the graphene and $\mathrm{AlGaN}$ semiconductor, the sample was baked at $200{ }^{\circ} \mathrm{C}$ for $3 \mathrm{~h}$ on a hot plate. Next, PMMA was removed by an acetone treatment at $60^{\circ} \mathrm{C}$. After a 100 -nm-thick $\mathrm{SiO}_{2}$ layer was deposited by a plasma-enhanced CVD system, an interdigitated finger structure was introduced by using a standard photolithographic technique and wet-etching of buffered oxide etchant. Probing metal electrodes of $\mathrm{Ni} / \mathrm{Au}$ were deposited by electron-beam evaporation and the graphene layer in unnecessary areas was etched away by $\mathrm{O}_{2}$ plasma.

Measurements. The I-V characteristics of the Gr/AlGaN/GaN photodetectors were measured by a source measurement unit (SMU, Keithley 4200) equipped with a dark-room probe station. The wavelength-dependent and temporal responsivities were measured by combining a Bentham switching monochromator and the SMU. The illumination power of the switching monochromator was calibrated by a Newport optical power meter. All presented measurements were taken at room temperature.

Received: 14 October 2020; Accepted: 4 December 2020

Published online: 16 December 2020

\section{References}

1. Chen, H., Liu, K., Hu, L., Al-Ghamdi, A. A. \& Fang, X. New concept ultraviolet photodetectors. Mater. Today 18, 493-502 (2015).

2. Tsao, J. Y. et al. Ultrawide-bandgap semiconductors: Research opportunities and challenges. Adv. Electron. Mater. 4, 1600501 (2018).

3. Chen, J., Ouyang, W., Yang, W., He, J. H. \& Fang, X. Recent progress of heterojunction ultraviolet photodetectors: Materials, integrations and applications. Adv. Funct. Mater. 30, 1909909 (2020).

4. Xie, C. et al. Recent progress in solar-blind deep-ultraviolet photodetectors based on inorganic ultrawide bandgap semiconductors. Adv. Funct. Mater. 29, 1806006 (2019).

5. He, T. et al. Broadband ultraviolet photodetector based on vertical $\mathrm{Ga}_{2} \mathrm{O}_{3} / \mathrm{GaN}$ nanowire array with high responsivity. Adv. Opt. Mater. 7, 1801563 (2019).

6. Zou, Y., Zhang, Y., Hu, Y. \& Gu, H. Ultraviolet detectors based on wide bandgap semiconductor nanowire: A review. Sensors 18, 2072 (2018).

7. Su, L., Yang, W., Cai, J., Chen, H. \& Fang, X. Self-powered ultraviolet photodetectors driven by built-in electric field. Small 13, 1701687 (2017).

8. Wang, Z. L. Self-powered nanosensors and nanosystem. Adv. Mater. 24, 280-285 (2012).

9. Munoz, E. et al. III nitrides and UV detection. J. Phys. Condens. Matter. 13, 7115-7137 (2001).

10. Sang, L., Liao, M. \& Sumiya, M. A comprehensive review of semiconductor ultraviolet photodetectors: From thin film to onedimensional nanostructures. Sensors 13, 10482-10518 (2013).

11. Razeghi, M. \& Rogalski, A. Semiconductor ultraviolet detectors. J. Appl. Phys. 79, 7433-7473 (1996).

12. Ambacher, O. et al. Two-dimensional electron gases induced by spontaneous and piezoelectric polarization charges in $\mathrm{N}$ - and Ga-face AlGaN/GaN heterostructures. J. Appl. Phys. 85, 3222 (1999).

13. Jang, H. W. et al. Mechanism of two-dimensional electron gas formation in $\mathrm{Al}_{\mathrm{x}} \mathrm{Ga}_{1-\mathrm{x}} \mathrm{N} / \mathrm{GaN}$ heterostructures. Appl. Phys. Lett. 81, 1249 (2002).

14. Ibbetson, J. P. et al. Polarization effects, surface states, and the source of electrons in AlGaN/GaN heterostructure field effect transistors. Appl. Phys. Lett. 77, 250 (2000).

15. Saito, W. et al. High breakdown voltage AlGaN-GaN power-HEMT design and high current density switching behavior. IEEE Trans. Electron Devices 50, 2528 (2003).

16. Jones, E. A., Wang, F. \& Costinett, D. Review of commercial GaN power devices and GaN-based converter design challenges. IEEE J. Emerg. Sel. Topics Power Electron. 4, 707-719 (2016).

17. Mayorov, A. S. et al. Micrometer-scale ballistic transport in encapsulated graphene at room temperature. Nano Lett. 11, 2396-2399 (2011).

18. Balandin, A. A. et al. Superior thermal conductivity of single-layer graphene. Nano Lett. 8, 902-907 (2008).

19. Nair, R. R. et al. Fine structure constant defines visual transparency of graphene. Science 320, 1308 (2008).

20. Lee, C., Wei, X., Kysar, J. W. \& Hone, J. Measurement of the elastic properties and intrinsic strength of monolayer graphene. Science 321, 385-388 (2008)

21. Tongay, S. et al. Graphene/GaN Schottky diodes: Stability at elevated temperatures. Appl. Phys. Lett. 99, 102102 (2011).

22. Pandit, B. \& Cho, J. AlGaN ultraviolet metal-semiconductor-metal with reduced graphene oxide contact. Appl. Sci. 8, 2098 (2018).

23. Pandit, B. \& Cho, J. Metal-semiconductor-metal ultraviolet photodiodes based on reduced graphene oxide/GaN Schottky contacts. Thin Solid Films 660, 824-827 (2018).

24. Monroy, E., Omnes, F. \& Calle, F. Wide-bandgap semiconductor ultraviolet photodetectors. Semicond. Sci. Technol. 18, R33-R51 (2003).

25. Carrano, J. C., Grudowski, P. A., Eiting, C. J., Dupuis, R. D. \& Campbell, J. C. Very low dark current metal-semiconductor-metal ultraviolet photodetectors fabricated on single-crystal GaN epitaxial layers. Appl. Phys. Lett. 70, 1992-1994 (1997).

26. Qi, L., Mok, K. R. C., Aminian, M., Charbon, E. \& Nanver, L. K. UV-sensitive low dark-count pureB single-photon avalanche diode. IEEE Trans. Electron. Devices 61, 3768-3774 (2014).

27. Ferrari, A. C. et al. Raman spectrum of graphene and graphene layers. Phys. Rev. Lett. 97, 187401 (2006).

28. Calizo, I., Bejenari, I., Rahman, M., Liu, G. \& Balandin, A. A. Ultraviolet Raman microscopy of single and multilayer graphene. J. Appl. Phys. 106, 043509 (2009). 
29. Wood, D. Optoelectronic Semiconductor Devices 249-275 (Prentice Hall, London, 1994).

30. Zhu, Y., Liu, K., Ai, Q., Hou, Q. \& Chen, X. A high performance self-powered ultraviolet photodetector based on a p-GaN/nZnMgO heterojunction. J. Mater. Chem. C 8, 2719-2724 (2020).

31. Jacopin, G. et al. Interplay of the photovoltaic and photoconductive operation modes in visible-blind photodetectors based on axial p-i-n junction GaN nanowires. Appl. Phys. Lett. 104, 023116 (2014).

32. Monroy, E. et al. Visible-blindness in photoconductive and photovoltaic AlGaN ultraviolet detectors. J. Electron Mater. 28, 240-245 (1999).

33. Xu, J. et al. High-gain $200 \mathrm{~ns}$ photodetectors from self-aligned CdS-CdSe core-shell nanowalls. Adv. Mater. 30, 1800413 (2018).

34. Zhai, T., Li, L., Fang, X., Bando, Y. \& Golberg, D. Recent developments in one-dimensional inorganic nanostructures for photodetectors. Adv. Funct. Mater. 20, 4233-4248 (2010).

35. Garg, M., Tak, B. R., Rao, V. R. \& Singh, R. Giant UV photoresponse of GaN-based photodetectors by surface modification using phenol-functionalized porphyrin organic molecules. ACS Appl. Mater. Interfaces 11, 12017-12026 (2019).

36. Martens, M. et al. High gain ultraviolet photodetectors based on AlGaN/GaN heterostructures for optical switching. Appl. Phys. Lett. 98, 211114 (2011)

37. Satterthwaite, P. F., Yalamarthy, A. S., Scandrette, N. A., Newaz, A. K. M. \& Senesky, D. G. High responsivity, low dark current ultraviolet photodetectors based on two-dimensional electron gas interdigitated transducers. ACS Photonics 5, 4277-4282 (2018).

38. Liu, J. M. Photonic Devices (Cambridge University Press, New York, 2005).

39. Wang, M.-Z. et al. $\mathrm{TiO}_{2}$ nanotube array/monolayer graphene film Schottky junction ultraviolet light photodetectors. Particle Particle Syst. Character. 30, 630-636 (2013).

40. Pandit, B., Seo, T. H., Ryu, B. D. \& Cho, J. Current transport mechanism in graphene/AlGaN/GaN heterostructures with various Al mole fractions. AIP Adv. 6, 065007 (2016).

\section{Acknowledgements}

The authors thank Dr. Young Jae Park for his assistance with sample preparation and gratefully acknowledge the financial support by the basic science research program through the National Research Foundation (NRF) of Korea funded by the Ministry of Education (NRF-2020R1I1A3066324).

\section{Author contributions}

B.P. and J.C. initiated the idea, fabricated the devices, and performed analysis. J.C. and E.F.S. contributed to the scientific discussion and wrote the main manuscript. All authors reviewed the manuscript.

\section{Competing interests}

The authors declare no competing interests.

\section{Additional information}

Supplementary Information The online version contains supplementary material available at https://doi. org/10.1038/s41598-020-79135-y.

Correspondence and requests for materials should be addressed to J.C.

Reprints and permissions information is available at www.nature.com/reprints.

Publisher's note Springer Nature remains neutral with regard to jurisdictional claims in published maps and institutional affiliations.

Open Access This article is licensed under a Creative Commons Attribution 4.0 International License, which permits use, sharing, adaptation, distribution and reproduction in any medium or format, as long as you give appropriate credit to the original author(s) and the source, provide a link to the Creative Commons licence, and indicate if changes were made. The images or other third party material in this article are included in the article's Creative Commons licence, unless indicated otherwise in a credit line to the material. If material is not included in the article's Creative Commons licence and your intended use is not permitted by statutory regulation or exceeds the permitted use, you will need to obtain permission directly from the copyright holder. To view a copy of this licence, visit http://creativecommons.org/licenses/by/4.0/.

(C) The Author(s) 2020 